\title{
Formação de Grupos em Ambientes Virtuais de Aprendizagem: Uma Revisão Sistemática da Literatura
}

\author{
Ilmara M. M. Ramos ${ }^{1,2}$, David B. Ramos ${ }^{1,2}$, Priscilla B. Nascimento1, Bruno F. \\ Gadelha $^{1}$, Elaine H. T. Oliveira ${ }^{1}$ \\ ${ }^{1}$ Instituto de Computação - Universidade Federal do Amazonas (UFAM) - Manaus - \\ AM - Brasil. \\ ${ }^{2}$ Instituto Federal de Educação, Ciência e Tecnologia do Amazonas (IFAM) - Campus \\ Parintins - Parintins - AM - Brasil. \\ \{ilmaramonteverde, david.ramos, \\ priscilla.batista, bruno, elaine\} @icomp.ufam.edu.br
}

\begin{abstract}
This paper presents an analysis of 19 articles, established through a Systematic Review of the Literature (RSL) in order to obtain information on how to build clusters for conducting collaborative activities in virtual environments, such as criteria, approaches, forms, type of data collection, algorithms and used virtual environments. The data obtained at the end of the RSL show that, predominantly, the criterion used was homogeneous, with selected approach, automatically collects implicit data using data mining algorithms and the Moodle platform for the formation of groups.
\end{abstract}

Resumo. Este artigo apresenta uma análise de 19 artigos, estabelecidos por meio de uma Revisão Sistemática da Literatura (RSL), a fim de obter informações de como se constroem os agrupamentos para a realização de atividades colaborativas em Ambientes Virtuais, tais como: critérios, abordagens, formas, tipo de coleta de dados, algoritmos e Ambientes Virtuais usados. Os dados obtidos ao final da RSL mostram que, predominantemente, o critério utilizado foi homogêneo, com abordagem selecionada, forma automática, coleta de dados implícita, usando algoritmos de mineração de dados e a plataforma Moodle para a formação de grupos.

\section{Introdução}

Existem na literatura Sistemas de Recomendação (SR) que tradicionalmente recomendam itens para indivíduos. Em alguns casos, entretanto, a recomendação para um grupo de indivíduos é mais apropriada, neste caso, primeiramente é necessário estabelecer os grupos. A partir deste contexto, o foco deste artigo são os sistemas de recomendação ou formação de grupos de aprendizes em ambientes virtuais de aprendizagem, com o objetivo de apresentar o resultado de uma revisão quasisistemática sobre essa temática. O intuito foi obter informações de como se constroem os agrupamentos para realizar atividades colaborativas em Ambientes Virtuais, quais critérios, abordagens, formas, tipo de coleta de dados, algoritmos, ambientes virtuais são usados para realizar a formação dos grupos. Os resultados mostram os principais dados obtidos por esta pesquisa, no que diz respeito à formação de grupos de aprendizes em ambientes virtuais de aprendizagem. Este artigo está estruturado da seguinte forma: a 
V Congresso Brasileiro de Informática na Educação (CBIE 2016)

Anais do XXVII Simpósio Brasileiro de Informática na Educação (SBIE 2016)

Seção 2 descreve o contexto da pesquisa, a Seção 3 define a metodologia utilizada; a Seção 4 como foi realizada a condução da revisão; a Seção 5 descreve análise dos resultados e, por fim, a Seção 6 com as considerações finais.

\section{Contexto da Pesquisa}

Nesta seção, serão abordadas as bases teóricas que darão suporte à pesquisa, buscando auxiliar a compreensão dos conceitos utilizados para subsidiar a sua construção. Serão apresentados os princípios teóricos sobre: Ambientes Virtuais de Aprendizagem e formação de Grupo para Aprendizagem Colaborativa. $\mathrm{Na} \mathrm{EaD}$ são utilizadas diversas plataformas de ensino, as quais nos permitem fazer alusão às salas de aula presenciais, possibilitando a aprendizagem aos alunos que delas se utilizam. Essas plataformas de ensino denominam-se Ambientes Virtuais de Aprendizagem (AVAs). Segundo Almeida, Vieira e Luciano (2001), os AVAs são ambientes que habitam o ciberespaço e incluem ferramentas que favorecem a comunicação de todos os envolvidos no processo de ensino-aprendizagem, oferecendo recursos que facilitem a aprendizagem individual e colaborativa. As plataformas irão auxiliar os estudantes nas suas interações virtuais com os recursos disponibilizados pelo professor e essas interações são importantes para o aprendizado tanto individual quanto em grupo.

\subsection{Formação de Grupo para Aprendizagem Colaborativa}

De acordo com Santos, Castro e Castro (2007), grupo é um pequeno conjunto de indivíduos que se reúnem por meio de uma determinada peculiaridade. Para que ocorra o agrupamento de indivíduos deve-se considerar diversas particularidades, como: abordagens, critérios, características e dados dos estudantes. Esses agrupamentos podem ser relevantes na aprendizagem individual e na colaborativa. Para Dillenbourg et al. (1996), a aprendizagem colaborativa consiste quando um grupo de pessoas se motivam em aprender algo juntas. Essa aprendizagem traz benefícios significativos ao processo de ensino-aprendizagem dos discentes, possibilitando a formação de grupos. A formação de grupos para aprendizagem colaborativa pode ser feita de duas formas, que segundo Citadin, Kemczinski e Matos (2014) se dividem em manual e automática. Manual quando o agrupamento é realizado fora do sistema, isto é, o professor realiza a formação do grupo de acordo com critérios estabelecidos por ele e posteriormente informa o grupo ao sistema. Automática quando o próprio sistema recomenda a formação do grupo de acordo com parâmetros e critérios estabelecidos anteriormente.

Para realizar o agrupamento dos aprendizes é necessário estabelecer qual será a abordagem de grupo utilizada. Na pesquisa realizada por Ounnas, Davis e Millard (2007) existem três tipos de abordagens para a formação de grupos que são: Randômico, Auto-Selecionado e Selecionado. Randômico são propostos pelo professor, este agrupa os discentes sem nenhum critério definido. Auto-Selecionado são grupos onde os alunos têm permissão para escolher o grupo ao qual querem pertencer. Selecionado é quando o professor escolhe os critérios para que o sistema realize a formação dos grupos. Também é preciso para formação de grupo estabelecer qual o tipo de coleta de dados que será utilizado para determinar as preferências dos usuários. Nessa perspectiva, Neto (2011), considera sob duas perspectivas a coleta de informações: Explícita e Implícita. Explícita são informações que são solicitadas aos usuários por meio de técnicas de elicitação de requisitos como, por exemplo, o questionário. Implícita: são informações 
V Congresso Brasileiro de Informática na Educação (CBIE 2016)

Anais do XXVII Simpósio Brasileiro de Informática na Educação (SBIE 2016)

que são extraídas a partir da navegação do usuário, sem que estes, tenham que fornecêlas diretamente, possibilitando inferir suas necessidades e preferências.

Além do tipo de coleta de dados, outro requisito a ser considerado é o tipo de classificação de grupo que será usado para realizar a formação de grupos. Na pesquisa de Citadin, Kemczinski e Matos (2014) as classificações dos grupos foram adaptadas para critérios, estes podem ser: Homogêneos, Heterogêneos e Ambos. Homogêneo é quando os membros dos grupos têm características semelhantes e Heterogêneo é quando os membros do grupo têm características diferentes para a formação do grupo. Ambos é quando permitir formar grupos tanto homogêneos quanto heterogêneos. Todas essas abordagens descritas acima, servirão de base para ajudar a compreender e responder as questões levantadas na RSL que é o foco deste artigo.

\section{Metodologia}

O estudo de uma RSL pode ser um fator preliminar de uma pesquisa, para se produzir um novo conhecimento, pois conforme Ramos et al. (2015), a RSL ajuda na descoberta de trabalhos relacionados a pesquisa e estes auxiliarão na construção de um novo conhecimento. Diante disso, pesquisas recentes têm se preocupado em realizar o levantamento do estado da arte relacionado com sistemas de recomendação ou formação de grupos tais como Cazella, Nunes e Reategui (2010), que descreve o estado da arte em sistemas de recomendação relatando um panorama geral sobre esse tema e descrevendo as técnicas e estratégias de recomendação usadas na literatura e Boratto e Carta (2010), que apresentam um levantamento do estado da arte sobre recomendação de grupo e expõe uma nova abordagem para identificação automática de grupos. Esta RSL está seguindo as orientações de Kitchenham e Chartes (2007) e constitui base fundamental deste artigo. Um estudo sobre recomendação de grupos ou formação de grupos em ambientes virtuais foi realizado por meio de uma RSL, cujo resultado está registrado em Ramos e Oliveira (2015). A revisão pode ser classificada como uma revisão quasisistemática, uma vez que não houve comparações entre publicações, bem como, uma pesquisa exploratória, preservando o mesmo formalismo e seguindo o mesmo processo da revisão sistemática Travassos et al. (2008). Durante o processo de revisão sistemática, utilizou-se uma ferramenta especialmente para este fim, chamada de StArt, esta foi desenvolvida na Universidade Federal de São Carlos, pelo Laboratório de Pesquisa em Engenharia de Software (LaPES). A versão 2.3.4.2 foi utilizada para auxiliar esta pesquisa.

\subsection{Objetivo e Questão da Pesquisa}

O objetivo da RSL foi identificar como são criados os sistemas de recomendação ou formação de grupos para atividades colaborativas dentro dos Ambientes Virtuais de Aprendizagem. A descrição do objetivo conforme o paradigma Goal-Question-Metric de Basili, Caldeiera e Rombach (1994), pode ser observada abaixo:

Analisar publicações científicas por meio de um estudo baseado em revisão sistemática. Com o propósito de identificar como são criados os sistemas de recomendação ou formação de grupos para atividades colaborativas usados na literatura voltados para Ambientes Virtuais de Aprendizagem. Com relação à agrupamento de alunos. Do ponto de vista dos pesquisadores, por meio do contexto acadêmico ou 
V Congresso Brasileiro de Informática na Educação (CBIE 2016)

Anais do XXVII Simpósio Brasileiro de Informática na Educação (SBIE 2016)

industrial. A RSL buscou responder a seguinte questão principal: Como se constroem os agrupamentos para realizar atividades colaborativas em Ambientes Virtuais?

\subsection{Método de Busca das Publicações}

Para realizar a busca da RSL foram utilizados os seguintes Mecanismos de Busca Acadêmica (MBA): IEEE Computer Science Digital Library e Scopus. A Scopus destaca-se por armazenar publicações de outras bibliotecas digitais. Além disso, Kitchenham e Chartes (2007), afirmam que a Scopus é a maior base de dados de indexação de resumos e citações, esta abrange uma visão sobre a produção científica do mundo nas áreas de ciência, tecnologia, medicina, ciências sociais, artes e humanidades.

\subsection{Expressão de Busca}

Para se chegar à expressão de busca utilizada na base da Scopus e IEEE foram realizados testes auxiliados com base em artigo de controle. O termo de busca usado nesta RSL está ilustrado a seguir no Quadro 1. Ela é composta por palavras-chave que representam a população (P), a intervenção (I). A composição básica da expressão de busca segue a seguinte estrutura: (P) AND (I). As palavras-chave da expressão de busca são apresentadas abaixo. Para a busca na Scopus, as aspas ("') foram substituídas por chaves $(\{\})$ sendo inicializada com TITLE-ABS-KEY.

\section{Quadro 1.Termo de busca}

\begin{tabular}{|l|}
\hline \multicolumn{1}{|c|}{ População } \\
\hline $\begin{array}{l}\text { "learning management system" OR "virtual learning environment" OR "moodle" OR "distance education" } \\
\text { OR "distance learning" OR "e-learning recommender system" }\end{array}$ \\
\hline \multicolumn{1}{|c|}{ Intervenção } \\
\hline $\begin{array}{l}\text { "clustering" OR "group recommendation" OR "group modeling" OR "group recommender systems" OR" } \\
\text { Profiles Aggregation" }\end{array}$ \\
\hline
\end{tabular}

\subsection{Critérios de Inclusão e Exclusão}

No Quadro 2, são apresentados os critérios de inclusão (CI) e exclusão (CE) utilizados durante a RSL. A seleção dos artigos pautou-se nos CI e CE abaixo relacionados e esses foram criados a partir dos artigos de controle.

\section{Quadro 2. Critérios de Inclusão e Exclusão}

(CI1) Artigos que apresentem representações de modelos, técnicas, processos, algoritmos de agrupamento sobre a formação de grupos de alunos em Ambientes Virtuais.

(CI2) Artigos que apresentem propostas de adaptação de trilhas de aprendizagem em Ambientes Virtuais.

\section{Critérios de Exclusão}

(CE1). Não serão selecionadas publicações em que as palavras-chave da busca não apareçam no título, resumo e/ou palavras-chaves.

(CE2) Não serão selecionadas publicações em que as siglas equivalentes não signifiquem: LMS "Learning Management System, VLE - "Virtual Learning Environment".

(CE3). Serão excluídas publicações que não tem disponibilidade de conteúdo para leitura (por exemplo, onde os trabalhos são pagos ou não disponibilizados pelas máquinas de buscas ou autores).

(CE4) Artigos que apenas apresentam relatos de experiência de algum modelo, técnica ou ferramenta.

(CE5) Artigos que apenas citam sistemas de e-learning (LMS, VLE, etc.), mas não o abordam como foco principal.

(CE6). Não serão selecionadas publicações que não relacionam formação de grupos e Ambientes Virtuais 
V Congresso Brasileiro de Informática na Educação (CBIE 2016)

Anais do XXVII Simpósio Brasileiro de Informática na Educação (SBIE 2016)

de Aprendizagem (LMS, CMS, ITS, LCMS).

(CE7). Não serão selecionadas publicações que apresentam Ambientes Virtuais de Aprendizagem, mas não descrevam formação de grupos ou recomendação de grupos.

(CE8). Não serão selecionadas publicações que descrevam formação de grupos ou recomendação de grupos, mas não relacionados a Ambientes Virtuais de Aprendizagem.

\subsection{Extração dos Dados}

Os dados extraídos durante a leitura dos artigos que foram aprovados no primeiro e segundo filtro seguiu uma lista de itens predefinidos conforme apresentado no Quadro 3. Os itens são: Pesquisa, campo referente ao número de artigos resultantes da RSL, Público Alvo, grupo de pessoas em que a pesquisa foi realizada, como por exemplo, alunos de graduação, alunos de idiomas, indústria/empresa, não informado (quando não for possível identificar na pesquisa), Forma, os tipos de formação de grupos podendo assumir as seguintes informações: automático, manual e outros, o campo Abordagem faz referências aos tipos de abordagens para se estabelecer os grupos que podem ser randômico, selecionado, auto-selecionado e outros, outro campo informado no quadro é o Critério, este descreve quais os tipos de agrupamento de alunos que deseja-se realizar podendo ser Heterogêneo, Homogêneo, Ambos e Outros. Outros dados relevantes extraído durante a execução da RSL foram quais os tipos de coleta de dados utilizados durante as pesquisas sobre agrupamentos e foram detectados os tipos explícitos, implícitos, ambos e outros, além também da identificação de quais Ambientes Virtuais que foram utilizados para a realização do agrupamento obtendo-se os Ambientes Moodle, MOOCs, WebClass-Rapsody e outros e por fim, os algoritmos utilizados na formação dos grupos podendo ter os seguintes valores Algoritmos Genéticos, Evolutivos, Mineração de dados, Ontologias e Outros. Este modelo foi baseado na taxonomia de Citadin, Kemczinski e Matos (2014). Todos os itens são de preenchimento obrigatório, exceto quando não informados ou quando não se aplicam à publicação.

Quadro 3. Extração dos Dados durante a Leitura dos Artigos

\begin{tabular}{|c|c|c|c|c|c|c|c|}
\hline Pesquisas & $\begin{array}{c}\text { Público } \\
\text { Alvo }\end{array}$ & Forma & Abordagem & Critério & $\begin{array}{c}\text { Tipo de } \\
\text { Coleta de } \\
\text { Dados }\end{array}$ & $\begin{array}{c}\text { Ambiente } \\
\text { Virtual } \\
\text { Utilizado }\end{array}$ & $\begin{array}{c}\text { Algo- } \\
\text { ritmo }\end{array}$ \\
\hline 1 & $\begin{array}{c}\text { Alunos de } \\
\text { Graduação }\end{array}$ & $\begin{array}{c}\text { Automá- } \\
\text { tico }\end{array}$ & Selecionado & Heterogêneo & Explícito & Moocs & Evolutivo \\
\hline 2 & $\begin{array}{c}\text { Alunos de } \\
\text { Graduação }\end{array}$ & Manual & Selecionado & Homogêneo & Explícito & Outros & Genético \\
\hline 3 & $\begin{array}{c}\text { Alunos de } \\
\text { Graduação }\end{array}$ & $\begin{array}{c}\text { Automá- } \\
\text { tico }\end{array}$ & Selecionado & Heterogêneo & Implícito & Moocs & Genético \\
\hline 4 & $\begin{array}{c}\text { Alunos de } \\
\text { Graduação }\end{array}$ & $\begin{array}{c}\text { Automá- } \\
\text { tico }\end{array}$ & Selecionado & Heterogêneo & Implícito & Moodle & $\begin{array}{c}\text { Data } \\
\text { Mining }\end{array}$ \\
\hline 5 & $\begin{array}{c}\text { Alunos de } \\
\text { Graduação }\end{array}$ & $\begin{array}{c}\text { Automá- } \\
\text { tico }\end{array}$ & Selecionado & Homogêneo & Explícito & Moodle & Evolutivo \\
\hline 6 & $\begin{array}{c}\text { Alunos de } \\
\text { Graduação }\end{array}$ & Ambos & Selecionado & Homogêneo & Ambos & Moodle & $\begin{array}{c}\text { Data } \\
\text { Mining }\end{array}$ \\
\hline 7 & $\begin{array}{c}\text { Alunos de } \\
\text { Graduação }\end{array}$ & Manual & Selecionado & Homogêneo & Implícito & Moodle & Ontologia \\
\hline 8 & $\begin{array}{l}\text { Não } \\
\text { informado }\end{array}$ & Manual & Selecionado & Homogêneo & Implícito & Moodle & $\begin{array}{c}\text { Data } \\
\text { Mining }\end{array}$ \\
\hline
\end{tabular}


V Congresso Brasileiro de Informática na Educação (CBIE 2016)

Anais do XXVII Simpósio Brasileiro de Informática na Educação (SBIE 2016)

\begin{tabular}{|c|c|c|c|c|c|c|c|}
\hline 9 & $\begin{array}{c}\text { Alunos de } \\
\text { Graduação }\end{array}$ & $\begin{array}{c}\text { Automá- } \\
\text { tico }\end{array}$ & Selecionado & Homogêneo & Implícito & Moodle & $\begin{array}{c}\text { Data } \\
\text { Mining }\end{array}$ \\
\hline 10 & $\begin{array}{c}\text { Indústria/ } \\
\text { Empresa }\end{array}$ & Manual & Selecionado & Homogêneo & Implícito & Moodle & Genético \\
\hline 11 & $\begin{array}{c}\text { Alunos de } \\
\text { Graduação }\end{array}$ & $\begin{array}{c}\text { Automá- } \\
\text { tico }\end{array}$ & Selecionado & Homogêneo & Implícito & Moodle & $\begin{array}{c}\text { Data } \\
\text { Mining }\end{array}$ \\
\hline 12 & $\begin{array}{c}\text { Não } \\
\text { informado }\end{array}$ & $\begin{array}{c}\text { Automá- } \\
\text { tico }\end{array}$ & Selecionado & Ambos & Ambos & Moodle & Genético \\
\hline 13 & $\begin{array}{c}\text { Não } \\
\text { informado }\end{array}$ & Manual & Selecionado & Homogêneo & Ambos & Outros & Outros \\
\hline 14 & $\begin{array}{c}\text { Não } \\
\text { informado }\end{array}$ & $\begin{array}{c}\text { Automá- } \\
\text { tico }\end{array}$ & Selecionado & Heterogêneo & Ambos & Outros & Evolutivo \\
\hline 15 & $\begin{array}{c}\text { Não } \\
\text { informado }\end{array}$ & $\begin{array}{c}\text { Automá- } \\
\text { tico }\end{array}$ & Selecionado & Homogêneo & Implícito & $\begin{array}{c}\text { WebClass- } \\
\text { Rapsody }\end{array}$ & $\begin{array}{c}\text { Data } \\
\text { Mining }\end{array}$ \\
\hline 16 & $\begin{array}{c}\text { Alunos de } \\
\text { Idiomas }\end{array}$ & $\begin{array}{c}\text { Automá- } \\
\text { tico }\end{array}$ & Selecionado & Outros & Explícito & Outros & Genético \\
\hline 17 & $\begin{array}{c}\text { Não } \\
\text { informado }\end{array}$ & Outros & Outros & Outros & Outros & Outros & Outros \\
\hline 18 & $\begin{array}{c}\text { Alunos de } \\
\text { Graduação }\end{array}$ & Manual & $\begin{array}{c}\text { Auto-Sele- } \\
\text { cionado }\end{array}$ & Outros & Explícito & Outro & Outros \\
\hline 19 & $\begin{array}{c}\text { Não } \\
\text { informado }\end{array}$ & $\begin{array}{c}\text { Automá- } \\
\text { tico }\end{array}$ & Selecionado & Homogêneo & Explícito & Outros & Evolutivo \\
\hline
\end{tabular}

\section{Condução da Revisão}

Finalizada a fase de planejamento da revisão, iniciou-se em novembro de 2015, a fase de condução da RSL, tendo como resultado da execução da string de busca 316 artigos, sendo 195 encontrados na biblioteca da Scopus e 121 artigos na IEEE. Estes artigos foram importados para a ferramenta StArt e após a importação foram identificados 35 artigos duplicados. Portanto, foram usados 281 artigos para análise do primeiro filtro. No primeiro filtro, foram analisados os 281 artigos por meio dos critérios de inclusão e exclusão, descritos na Seção 3.4. A análise de cada artigo foi de acordo com cada critério, conforme ilustrado na Figura 1.

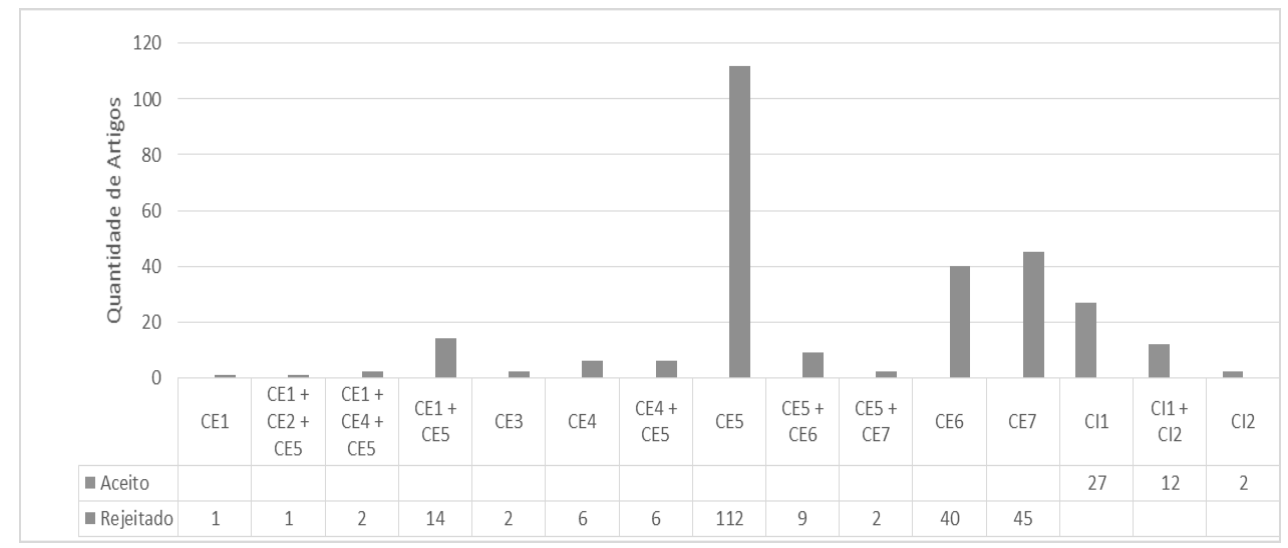

Figura 1. Resultado do primeiro filtro de acordo com os critérios de Inclusão e Exclusão.

Para isso, o título, resumo/abstract e as palavras-chave de cada publicação foram analisadas, para verificar quais artigos são úteis para o contexto da pesquisa. Após execução do primeiro filtro, 41 artigos foram aceitos para execução do segundo filtro e 
V Congresso Brasileiro de Informática na Educação (CBIE 2016)

Anais do XXVII Simpósio Brasileiro de Informática na Educação (SBIE 2016)

240 rejeitados. No processo de execução do segundo filtro, foram analisados os artigos aceitos onde estes foram lidos na íntegra e analisados conforme os critérios da Seção 3.4. Após a execução do segundo filtro, obteve-se como resultado 19 artigos rejeitados, 3 duplicados e 19 aceitos, conforme ilustrado na Figura 2.

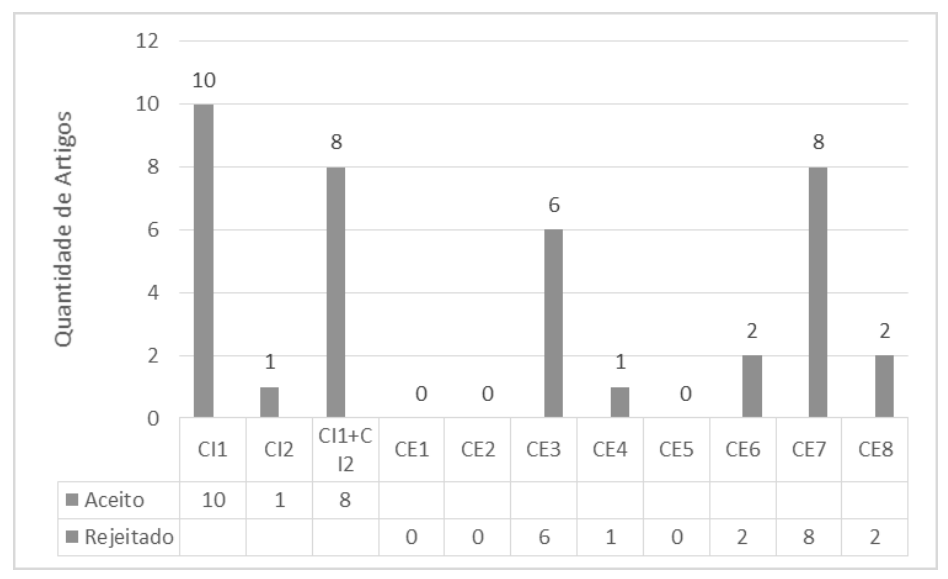

Figura 2. Resultado do segundo filtro de acordo com os critérios de Inclusão e Exclusão.

\section{Análise e Discussão dos Dados}

Na RSL, a questão principal era saber como se constroem os agrupamentos para realizar as atividades de aprendizagem em Ambientes Virtuais. Ao final da RSL, foram identificadas 19 publicações pertinentes. Foi percebida, na análise dos resultados da revisão, que para a formação de grupos de aprendizes as coletas de dados, para se extrair as informações sobre as preferências dos discentes, é feita 33\% de forma explícita, $45 \%$ de forma implícita por meio dos logs de acesso dos usuários no Ambiente Virtual e 22\% por ambos, ou seja, utilizam tanto a coleta de dados implícita quanto explícita, o resultado da análise está ilustrado na Figura 3. Outra análise realizada foi referente quanto à forma usada para realizar a formação dos grupos nos Ambientes Virtuais, constatou-se que 58\% das publicações foram de maneira automática, 32\% manual, 5\% ambos, isto é, adotaram ambas as formas para a formação e 5\% Outros (não foi possível classificar na publicação qual forma foi utilizada), conforme ilustrado na Figura 3.
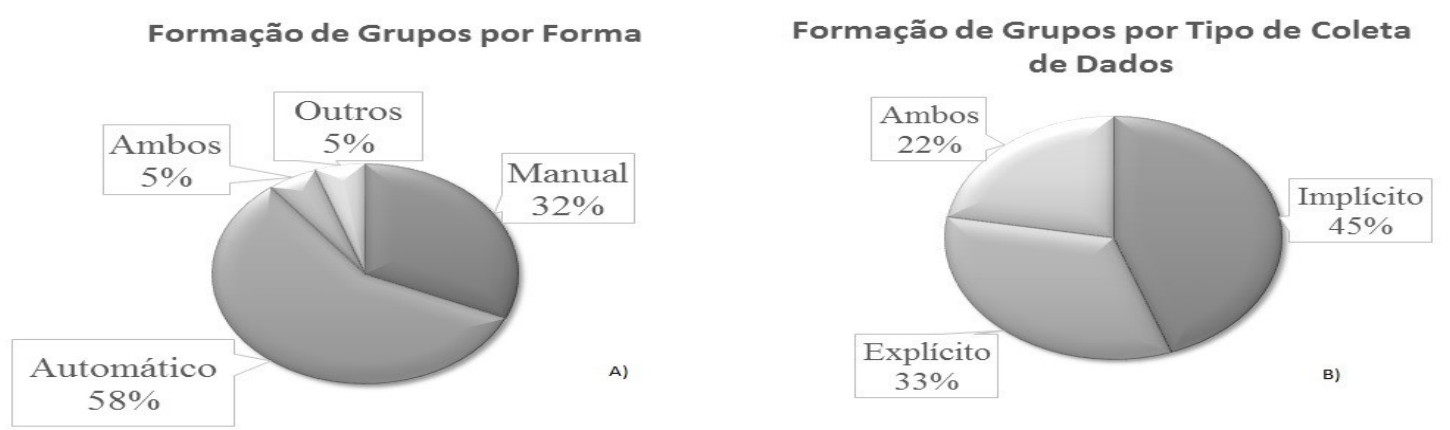

Figura 3. Resultado da Análise de Formação de Grupos por forma e Tipo de Coleta.

Em relação aos critérios utilizados para formação, 58\% foram homogêneos, $21 \%$ heterogêneo, $16 \%$ Outros (não foi possível classificar qual critério que foi 
V Congresso Brasileiro de Informática na Educação (CBIE 2016)

Anais do XXVII Simpósio Brasileiro de Informática na Educação (SBIE 2016)

utilizado) e 5\% ambos, conforme ilustrado na Figura 4. Pode-se observar que $90 \%$ das publicações utilizam a abordagem Selecionado, 5\% Auto-Selecionado e 5\% Outros (não foi possível classificar), conforme ilustrado na Figura 4.
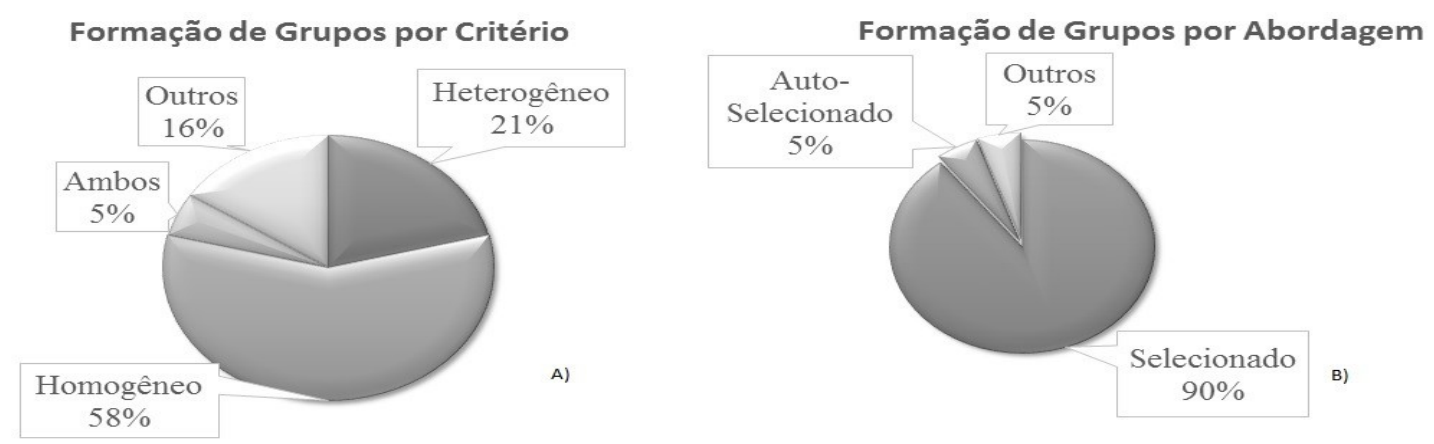

Figura 4. Resultado da Análise de Formação de Grupos por Critério e Abordagem.

Outra análise feita nesse artigo foi em relação aos AVAs usados na realização do agrupamento dos discentes e os algoritmos utilizados para a formação dos grupos. A Figura 5 ilustra a relação dos algoritmos e os Ambientes Virtuais, sendo representado nas linhas verticais do gráfico, os algoritmos usados e nas linhas horizontais os AVAs usados para formação dos grupos. O item Outros corresponde a Ambientes Virtuais criados pela própria universidade.

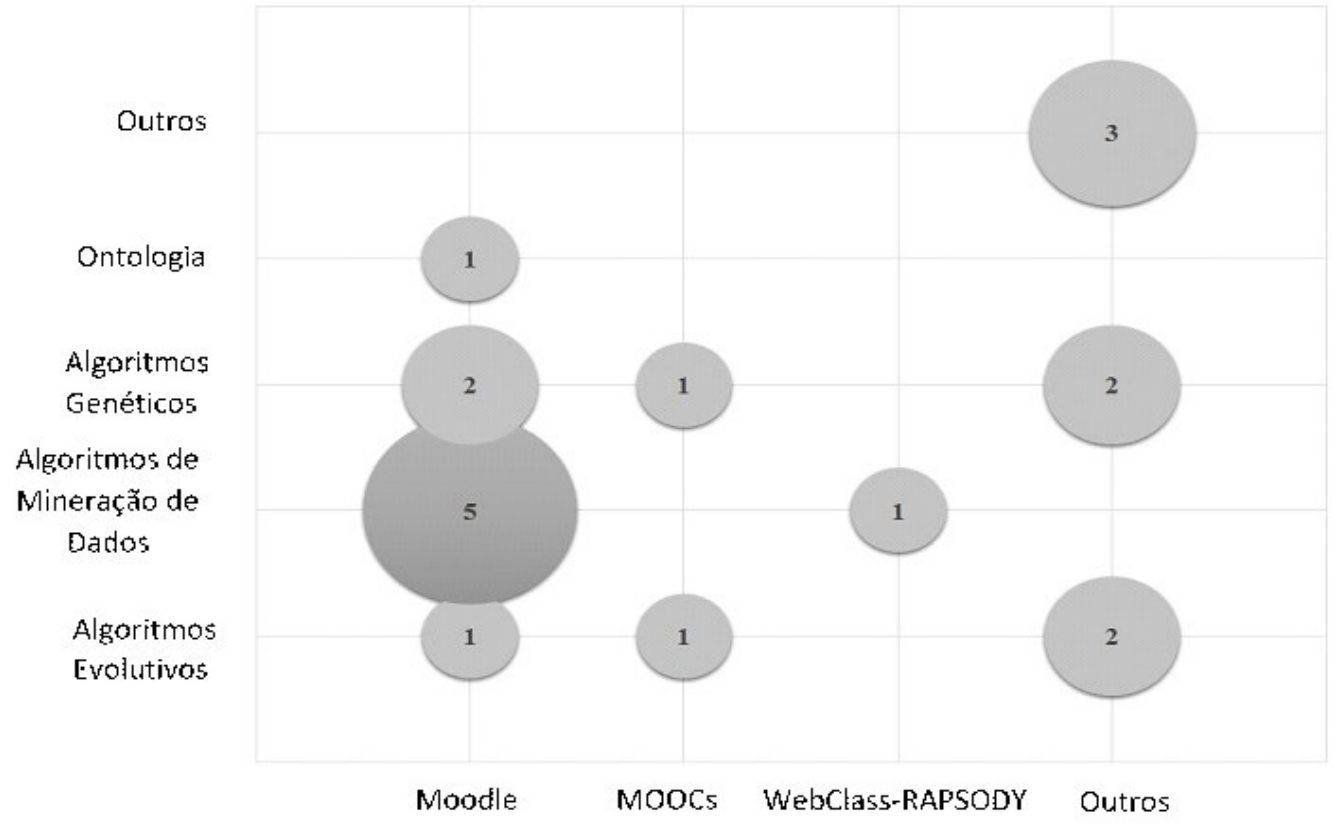

Figura 5. Relação algoritmos x Ambientes Virtuais de Aprendizagem

A análise mostra que o Ambiente Virtual mais utilizado nas pesquisas é o Moodle e que os algoritmos de Mineração de dados são os mais usados para a formação de grupos nos AVAs. Para realização desta RSL, o ano de publicação não foi delimitado, sendo assim, os artigos selecionados após os filtros foram publicados entre os anos de 2002 a 2015. A Figura 6, mostra a evolução das pesquisas selecionadas, que utilizam formação de grupos em AVAs ao longo dos anos. A distribuição temporal mostrou que apesar de não obter um número expressivo de publicações selecionadas por 
V Congresso Brasileiro de Informática na Educação (CBIE 2016)

Anais do XXVII Simpósio Brasileiro de Informática na Educação (SBIE 2016)

ano, houve um aumento nas publicações entre os anos de 2009 e 2012, o que demonstra o interesse dos pesquisadores em trabalhos de formação de grupos em AVAs.

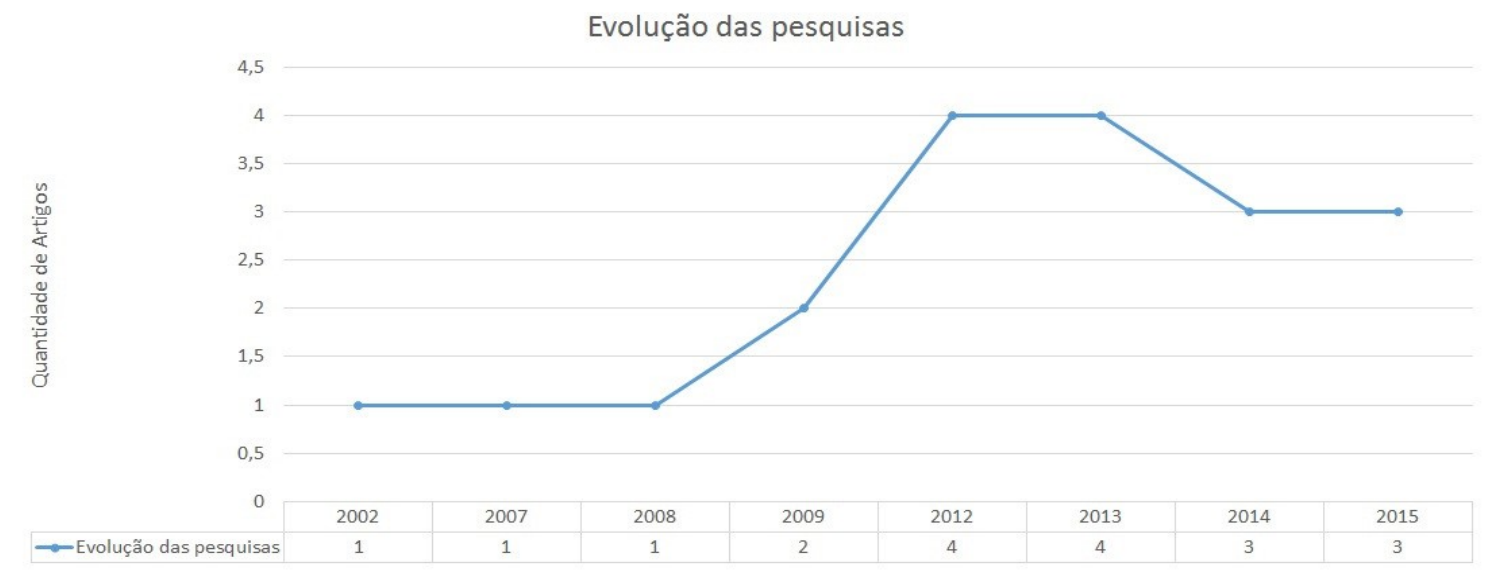

Figura 6.Evolução das pesquisas sobre formação de grupos em Ambientes Virtuais.

\section{Considerações Finais}

Este artigo apresentou os dados produzidos durante a RSL sobre o estado da arte de trabalhos relacionados a sistemas de recomendação ou a formação de grupos de aprendizes em AVAs. A análise foi focada em aspectos chaves para maior obtenção de informações, com o objetivo de identificar de que forma os trabalhos tem direcionado suas soluções, verificou-se com o resultado obtido que as publicações usam a abordagem selecionada, a forma automática, o tipo de coleta de dados implícito por meio dos logs de acesso da plataforma, o critério homogêneo, os algoritmos utilizados foram de mineração de dados e a plataforma Moodle. Espera-se que este trabalho possa contribuir por reunir os aspectos que a área apresenta e como esta linha de pesquisa é apresentada atualmente na literatura. Como próximos passos desta pesquisa, pretende-se definir aspectos para o desenvolvimento de um modelo de recomendação de grupos para atividades colaborativas utilizando a caracterização dos aprendizes baseadas em trilhas de aprendizagem apresentados na pesquisa de Ramos, Oliveira e Ramos (2015).

\section{Agradecimentos}

Parte dos resultados apresentados neste artigo foram obtidos através de atividades de P\&D do projeto PROMOBILE patrocinado pela Samsung Eletrônica da Amazônia Ltda nos termos da lei federal brasileira $n^{\circ}$ 8.248/91. Agradecemos também à FAPEAM pelo suporte financeiro concedido para a realização do projeto ao qual o artigo pertence. E ao 'MobMoodle: um ambiente para suporte à aprendizagem móvel', Edital FAPEAM 016/2013 ProTI-Pesquisa.

\section{Referências}

Almeida, C., Vieira, M. e Luciano, N. (2001) “Ambiente Virtual de Aprendizagem: Uma proposta para autonomia e cooperação na disciplina de informática”, In: XII Simpósio Brasileiro de Informática na Educação (SBIE 2001) , 12, p.431-439. Espírito Santo. 
V Congresso Brasileiro de Informática na Educação (CBIE 2016)

Anais do XXVII Simpósio Brasileiro de Informática na Educação (SBIE 2016)

Basili, V., Caldiera, G. e Rombach, H. (1994) “The Experience Factory”. Encyclopedia of Software Engineering. New York: John Wiley \& Sons.

Boratto, L. e Carta, S. (2010) "State-of-the-art in Group Recommendation and new approaches for Automatic Identification of Groups". Studies in Computational Intelligence, v.324, p.1-20.

Cazella, S., Nunes, M. e Reategui, E. (2010) "A ciência da Opinião: Estado da arte em Sistemas de Recomendação", In: XXX Congresso da Sociedade Brasileira de Computação (SBC) Jornada de Atualização de Informática (JAI), p.161-216.

Citadin, J., Kemczinski, A. e Matos, A. (2014) "Formação de Grupos para Aprendizagem Colaborativa: Um Mapeamento Sistemático da Literatura", In: XIX Conferência Internacional sobre Informática na Educação, 19,v. 10,p. 46-54.

Dillenbourg, P., Baker, M. Blaye, A. e Malley, C. (1996) "The Evolution of Research on Collaborative Learning”, In: Spada, E., Reiman, P.(eds) Learning in Humans and Machine: Towards an Interdisciplinary Learning Science, p.189-211. Elsevier, Oxford.

Kitchenham, B. e Chartes, S. (2007) "Guidelines for performing Systematic Literature Reviews in Software Engineering” EBSE Technical Report EBSE-2007-01, Software Engineering Group Department of Computer Science Keele University.

Neto, M. (2011) "Estratégia Híbrida para Recomendação Personalizada utilizando o Guia de Programação Eletrônico".106p. Dissertação (Mestrado Acadêmico em Ciência da Computação), Universidade Estadual do Ceará, Fortaleza.

Ounnas, A., Davis, H. e Millard, D. (2007) "Towards Semantic Group Formation", In: Proceedings of The 7th IEEE International Conference on Advanced Learning Technologies (ICALT 2007), p. 825-827.

Ramos, I. e Oliveira, E. (2015) "Relatório Técnico da Revisão Sistemática de Literatura sobre Recomendação de Grupos para Atividades Colaborativas em Ambientes Virtuais de Aprendizagem". Universidade Federal do Amazonas. Manaus. http://mobmoodle.icomp.ufam.edu.br/projeto/?, Dezembro.

Ramos, I., Oliveira, E. e Ramos, D. (2015) "Recomendação de grupos para atividades colaborativas utilizando a caracterização dos aprendizes baseada em trilhas de aprendizagem”. In: Simpósio Brasileiro de Sistemas Colaborativos (SBSC), p. 213219, Salvador.

Ramos, D., Oliveira, E. Ramos, I. e Oliveira K. (2015) "Trilhas de Aprendizagem em Ambientes Virtuais de Ensino-aprendizagem: Uma Revisão Sistemática da Literatura", In: XXVI Simpósio Brasileiro de Informática na Educação (SBIE 2015), 26, p.338-347.Maceió.

Santos, S., Castro, J. e Castro, T. (2007) "Alteração do Modelo de Grupos do Moodle para apoiar a Colaboração", In: Anais do XVIII Simpósio Brasileiro de Informática na Educação (SBIE 2007), 18, p.123-134. Porto Alegre.

Travassos, G., Dos Santos, P. e Neto, P. (2008) “An Environment to Support Large Scale Experimentation in Software Engineering", In: Proceedings of 13th IEEE International Conference on the Engineering of Complex Computer Systems (ICECCS), p. 193-202, Belfast, United Kingdom. 